\title{
Posterior Circulation Aneurysms: A Critical Appraisal of a Surgical Series in Endovascular Era
}

\author{
Sabino Luzzi, Mattia Del Maestro, and Renato Galzio
}

\section{Introduction}

Posterior circulation aneurysms have a worse natural history than anterior aneurysms, mainly because of their higher risk of rupture and poor outcome [1]. This aspect imposes the need for treatment in most cases, especially in younger patients. The advent of the endovascular era and its constant refinement through the continuous improvement of the devices has dramatically changed the treatment standard for many but not all of the posterior circle aneurysms. Exceptions are aneurysms involving the distal segments of the cerebellar arteries, most basilar tip aneurysms, and the giant ones for which microneurosurgery remains a rational option. The aim of this study is a critical appraisal of the overall results of a retrospective surgical series aimed to identify those posterior circulation aneurysms for which microneurosurgery still today maintains a key role.
S. Luzzi $(\bowtie) \cdot R$. Galzio

Neurosurgery Unit, Department of Clinical-Surgical, Diagnostic and Pediatric Sciences, University of Pavia, Pavia, Italy

Neurosurgery Unit, Department of Surgical Sciences, Fondazione IRCCS Policlinico San Matteo, Pavia, Italy

e-mail: sabino.luzzi@unipv.it

\section{Del Maestro}

Neurosurgery Unit, Department of Surgical Sciences, Fondazione IRCCS Policlinico San Matteo, Pavia, Italy

$\mathrm{PhD}$ School in Experimental Medicine, Department of ClinicalSurgical, Diagnostic and Pediatric Sciences, University of Pavia, Pavia, Italy

e-mail: m.delmaestro@smatteo.pv.it

\section{Materials and Methods}

Collected data concerned demographics, clinical onset, the prevalence of site and size, approaches, and outcome of 149 patients surgically treated because they harbored one or more posterior circulation aneurysms have been retrospectively reviewed. All the patients were operated on by the senior author $(\mathrm{RG})$ in three different hospitals over a period of 28 years between January 1990 and December 2018. Aneurysms were classified as proximal and distal. The proximal ones involved vertebral artery (VA), basilar artery (BA), and the proximal segments of the posterior cerebral artery (PCA) and cerebellar arteries. The remaining sites were considered as distal. For outcome evaluation, the patients were divided in to two groups: $<65$ and $\geq 65$ years old. The Angiographic outcome was evaluated based on the complete exclusion of the aneurysm at a six-month follow-up. The Neurological overall outcome was reported according to patients' age, clinical onset, and site, and size of the aneurysms. Glasgow Outcome Score (GOS) 1 and 2 were considered as "good recovery," whereas GOS 3, 4, and 5 were considered as "moderate disability," "severe disability," and "death-vegetative state," respectively.

\section{Results}

\section{Patients Demographics and Clinical Data}

Average patient age was $56.7 \pm 14.2$ years. Admission contrast-enhanced computed tomography (CT) angiography was the rule for all patients. Preoperative digital subtraction angiography (DSA) was performed in all unruptured or complex aneurysms. In elective cases, the need for a balloon test occlusion (BTO) was assessed on a case-by-case basis. A contrast-enhanced MRI was performed in all very large and giant aneurysms to reveal 
Table 1 Site and Size Prevalence of Posterior Circulation Aneurysms

\begin{tabular}{|c|c|c|c|c|c|c|}
\hline \multirow[b]{2}{*}{ Site } & & \multirow[t]{2}{*}{$N(\%)$} & \multicolumn{4}{|l|}{ Size } \\
\hline & & & $\begin{array}{l}\text { Small }(7 \mathrm{~mm}) \\
N(\%)\end{array}$ & $\begin{array}{l}\text { Regular } \\
(7-12 \mathrm{~mm}) \\
N(\%)\end{array}$ & $\begin{array}{l}\text { Large }(13-24 \mathrm{~mm}) \\
N(\%)\end{array}$ & $\begin{array}{l}\text { Giant } \\
(25 \mathrm{~mm}) \\
N(\%)\end{array}$ \\
\hline \multirow[t]{4}{*}{ Proximal } & Basilar tip + PCA (P1) + SCA & $91(58 \%)$ & $17(18.7 \%)$ & $57(62.6 \%)$ & $7(7.7 \%)$ & $10(11 \%)$ \\
\hline & Midbasilar trunk + proximal AICA & $9(5.7 \%)$ & $2(22.2 \%)$ & $4(44.4 \%)$ & $2(22.2 \%)$ & $1(11.1 \%)$ \\
\hline & VB Junction & $10(6.4 \%)$ & $2(20 \%)$ & $5(50 \%)$ & $2(20 \%)$ & $1(10 \%)$ \\
\hline & $\begin{array}{l}\text { VA (V4) + proximal PICA (anterior + lateral } \\
\text { medullary segment) }\end{array}$ & $27(17.2 \%)$ & $5(18.5 \%)$ & $16(59.3 \%)$ & $3(11.1 \%)$ & $3(11.1 \%)$ \\
\hline \multirow[t]{4}{*}{ Distal } & PCA (P2-P3) & $7(4.5 \%)$ & $1(14.3 \%)$ & $2(28.6 \%)$ & $1(14.3 \%)$ & $3(42.9 \%)$ \\
\hline & Distal SCA & $3(1.9 \%)$ & - & $1(33.3 \%)$ & $1(33.3 \%)$ & $1(33.3 \%)$ \\
\hline & Distal AICA & $4(2.5 \%)$ & $2(50 \%)$ & $1(25 \%)$ & - & $1(25 \%)$ \\
\hline & $\begin{array}{l}\text { Distal PICA (tonsillo-medullary } \\
+ \text { telovelotonsillar + cortical segment) }\end{array}$ & $6(3.8 \%)$ & - & $2(33.3 \%)$ & $2(33.3 \%)$ & $2(33.3 \%)$ \\
\hline \multicolumn{2}{|r|}{ 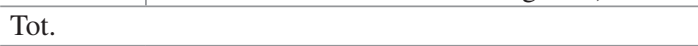 } & 157 & $29(18.5 \%)$ & $88(56.1 \%)$ & $18(11.5 \%)$ & $22(14 \%)$ \\
\hline
\end{tabular}

$P C A(P 1)$ proximal segment of the posterior cerebral artery, SCA superior cerebellar artery, AICA anterior inferior cerebellar artery, $V B$ junction, vertebro-basilar junction, $V A(V 4)$ intradural segment of the vertebral artery, $P I C A$ posterior inferior cerebellar artery, $P C A(P 2-P 3)$ distal segments of the posterior cerebral artery

eventual intraluminal thromboses. In 98 patients, a hemorrhagic onset occurred. The Average admission Hunt-Hess score was $2.17 \pm 0.8$ and the mean Fisher grade was $1.9 \pm 0.8$. One hundred thirty-seven aneurysms were classified as proximal and 20 as distal. Table 1 reports the prevalence of proximal and distal posterior circulation aneurysms according to site and size (Table 1). About ruptured aneurysms - apart from rare cases of young patients having an impending life hematoma, for whom the indication for surgery was based mainly upon an evidence-based management algorithm about intracerebral hemorrhages reported by our group [2] - mainly patients with an admission Hunt-Hess score of 1-3 underwent surgery. In $92 \%$ of cases, an early surgery (within $24 \mathrm{~h}$ ) was performed. One hundred fifty-seven aneurysms were consecutively operated on; six patients had two aneurysms and one patient harbored three different aneurysms. In three cases, two different procedures were performed on the same patient. A total of 152 procedures were performed.

\section{Surgery}

\section{Approach Selection}

Approaches were selected according to site and angioarchitecture. Pterional and cranio-orbitary approaches were utilized to basilar tip, proximal (P1) PCA, and superior cerebellar artery (SCA). For these aneurysms, pterional approach was usually "extended" to comprehend wide drilling of the lesser sphenoid wing, a large opening of the sylvian fissure, an extradural or intradural anterior clinoidectomy, and an intradural posterior clinoidectomy. Cranioorbitary corridors were preferentially employed in large and giant aneurysms. Al-Mefty's combined petrosal approach [3] was employed for the midbasilar trunk and proximal anterior inferior cerebella artery (AICA). The Farlateral retrocondylar approach was the corridor of choice for aneurysms involving VB junction, VA, and the proximal PICA, although the transcondylar variant was rarely necessary. Regarding the distal localizations, a subtemporal trans-tentorial approach was commonly used to treat P2-P3 PCA aneurysms. Conversely, aneurysms involving the distal segments of AICA, SCA, and PICA were elegantly treated by a retrosigmoid route.

\section{Direct vs. Indirect Treatment}

In all but three aneurysms a direct treatment was possible. A total of 128 aneurysms were successfully clipped. Trapping was the solution to aneurysms that were not amenable for clipping, under two conditions: if the patient tolerated the BTO, and the aneurysms were far distal having no need for revascularization. In one elective case of complex giant posterior projecting basilar tip aneurysm, an extracranial to intracranial (EC-IC) occipital artery (OA) — right P3 PCA bypass, with a radial artery graft, was performed. In two other elective patients, an intracranial to intracranial (IC-IC) PICA-PICA in situ bypass was carried out preceding, in both cases, the trapping of a complex VB junction aneurysm. Table 2 reports the types of treatment and the surgical techniques comprehensively employed in the current series (Table 2).

\section{Temporary Clipping and Neurophysiological Monitoring}

In the present series, the anesthesia protocol used by our group was specifically designed to allow the intraoperative neurophysiological monitoring during neurovascular surgery 
Table 2 Type and Prevalence of the Employed Surgical Techniques

\begin{tabular}{l|l|l}
\hline Type of treatment & Technique & $N(\%)$ \\
\hline \multirow{2}{*}{ Direct } & Clipping & $128(81.5 \%)$ \\
\cline { 2 - 3 } & Trapping & $19(12.1 \%)$ \\
\cline { 2 - 3 } & Wrapping & $7(4.5 \%)$ \\
\hline \multirow{2}{*}{ Indirect } & OA-P3 PCA bypass & $1(0.6 \%)$ \\
\cline { 2 - 3 } & PICA-PICA bypass & $2(1.3 \%)$ \\
\hline Tot. & & 157
\end{tabular}

$O A$ occipital artery, P3 PCA P3 segment of the posterior cerebral artery, $P I C A-P I C A$ posterior-inferior cerebellar artery to posterior-inferior cerebellar artery side to side in situ by pass

and was the same as employed for brain arteriovenous malformations and giant aneurysms in general [4-13]. A combined somatosensory-motor-brainstem auditory evoked potentials and EEG-based intraoperative neurophysiological monitoring were implemented in 2012. Neurophysiological Monitoring was performed in all proximal or complex aneurysms electively treated.

\section{Technological Adjuvants and Flow Assessment Techniques}

Neuronavigation and endoscope-assisted techniques were commonly employed, the latter being useful for both the aneurysms having a huge blind spot and those very close to perforating arteries. Since 2007, a micro-Doppler $(20 \mathrm{MHz}$ System, Mizuho Medical Co., Ltd., Tokyo, Japan) ultrasoundbased evaluation of the flow was implemented. Indocyanine green video angiography (Flow 800 Infrared Module, OPMI Pentero 800, Zeiss, Oberkochen, Germany) and fluorescein angiography (Yellow 560 Fluorescence Module, Kinevo 900, Zeiss, Oberkochen, Germany) were introduced in 2009 and 2018, respectively.

\section{Neurological Outcome}

The best outcome was achieved in patients $<65$ years old harboring an unruptured aneurysm. Table 3 reports the overall outcome according to the clinical onset and patient age (Table 3). The best results were also observed in small-toregular aneurysms involving basilar tip, distal branches of the cerebellar arteries, VA, and the proximal PICA. Figures 1 and 2 report the overall patient outcomes in proximal and distal aneurysms, respectively, according to their site and size (Figs. 1 and 2).

\section{Angiographic Outcome}

Six-month postoperative angiography was available in all but 17 patients. If no remnants were revealed at the first postoperative angiography, patients underwent a CT angiography for further annual follow-ups.

In $88.5 \%$ of patients, total exclusion of the aneurysms was achieved using a single procedure. In three cases, a remnant was revealed, causing a redo surgery. Along with an average follow-up of $67.1 \pm 61$ months, no recurrences occurred.

\section{Illustrative Case}

The case of a left giant VA-PICA aneurysm is reported (Fig. 3). A 42-year-old patient suffering from long-lasting dizziness underwent a contrast-enhanced MRI that showed a left giant VA causing a brainstem compression (Fig. 3a). CT angiography and DSA demonstrated the involvement of PICA (Fig. 3b, c). No crossflow was revealed by BTO (Fig. 3d). A left far-lateral retrocondylar approach was performed and the aneurysm was clipped using stacking-seating technique (Fig. 3e, f). Postoperative DSA documented the complete exclusion of the aneurysm with a preserved flow into the left PICA (Fig. 3g). The patient had a good recovery (GOS 5).

Table 3 Patients' overall outcome according to the clinical onset and the patients' age

\begin{tabular}{l|l|l|l|l}
\hline \multirow{2}{*}{\begin{tabular}{l} 
Outcome \\
\cline { 2 - 5 }
\end{tabular}} & Clinical onset & Pon-hemorrhagic & $<65$ years-old & $\geq 65$ years-old \\
\hline $\begin{array}{l}\text { Good Recovery } \\
\text { [No. of patients (\%)] }\end{array}$ & $80(81.6 \%)$ & $45(88.2 \%)$ & $66(83.5 \%)$ & $33(47.1 \%)$ \\
\hline $\begin{array}{l}\text { Moderate disability } \\
\text { [No. of patients (\%)] }\end{array}$ & $4(4.1 \%)$ & $2(3.9 \%)$ & $8(10.1 \%)$ & $14(20 \%)$ \\
$\begin{array}{l}\text { Severe disability } \\
{[\text { No. of patients }(\%)]}\end{array}$ & $3(3.1 \%)$ & $1(2 \%)$ & $1(1.3 \%)$ & $9(12.9 \%)$ \\
\hline $\begin{array}{l}\text { Death-vegetative state } \\
\text { [No. of patients }(\%)]\end{array}$ & $11(11.2 \%)$ & $3(5.9 \%)$ & $4(5.1 \%)$ & $14(20 \%)$ \\
\hline
\end{tabular}



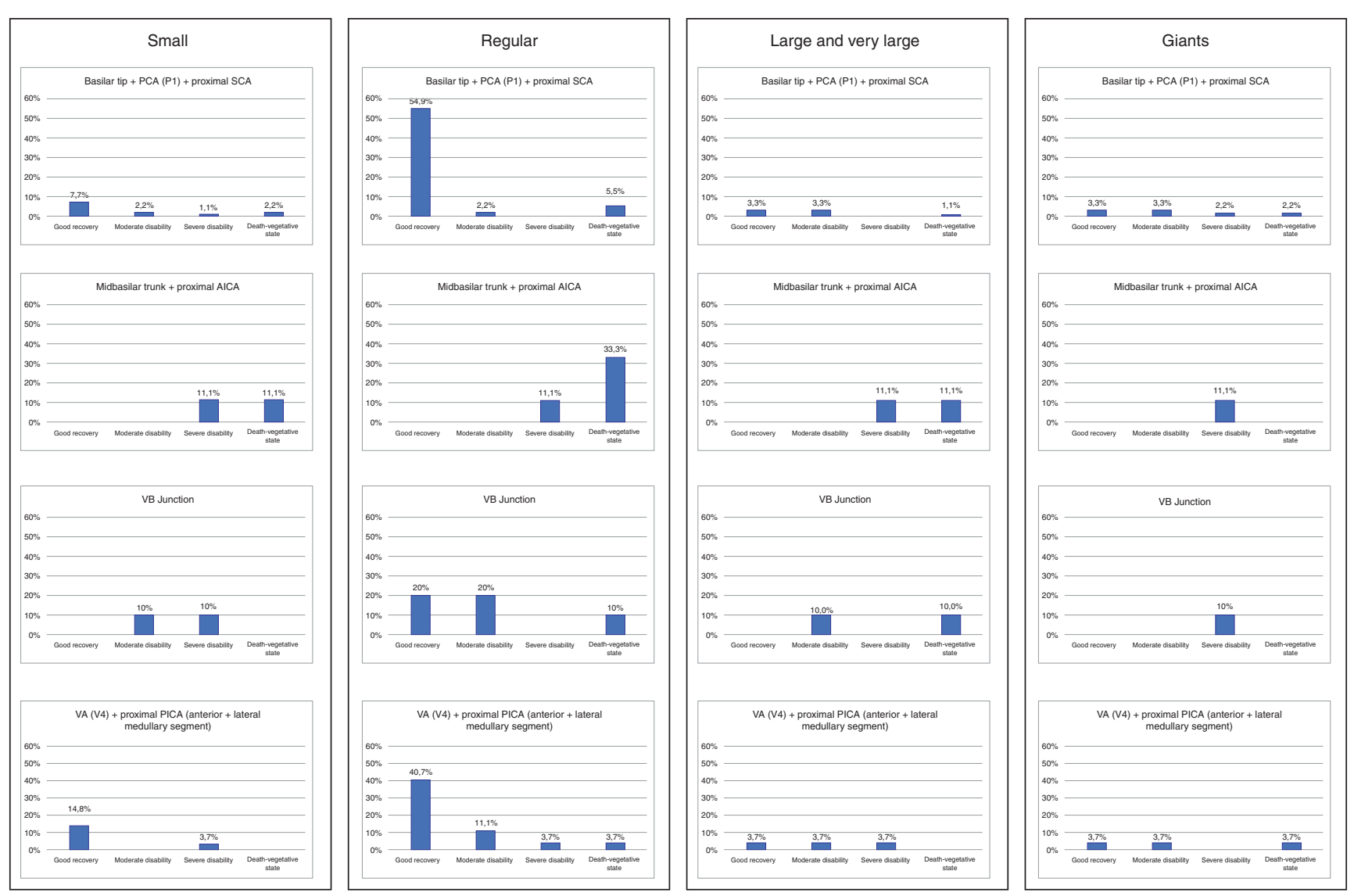

Fig. 1 Bar graph showing the overall patient outcome in proximal aneurysms according their site and size

\section{Discussion}

Although not free from possible complications, neuroendovascular techniques have reached a level of effectiveness that certainly makes them suitable for a large part of posterior circulation aneurysms. Conversely, microneurosurgery still has a very important role in the treatment of aneurysms involving distal segments of the cerebellar arteries, giant aneurysms, a large number of aneurysms affecting basilar tip and proximal SCA, an equally large part of VA-PICA aneurysms and, more generally, complex aneurysms not amenable to endovascular treatment [6, 14-22]. Particularly, the well-established role that microneurosurgery plays in the treatment of distal infratentorial aneurysms is the same, in terms of durability, as its role in treating distal supratentorial ones [23].
The present retrospective series also confirms these data, suggesting that microneurosurgery should be considered as the treatment of choice especially for elective patients younger than 65 years old. Some technical aspects as follow ought to be considered to achieve the best results: First, a detailed static and dynamic preoperative evaluation of the aneurysm's angioarchitecture and the flowrelated aspects are both imperative to plan the treatment strategy. A 4- or 6-vessel DSA, depending on the need for flow replacement, is recommended for all the posterior circulation aneurysms, and also addresses the wellknown risk of missing very distal PICA ones [24]. Indeed, one of the authors reported a very rare case of an extracranial small aneurysm of the PICA which was initially missed by CT angiography. Second, a careful preoperative evaluation of the patient's vascular and bony anat- 

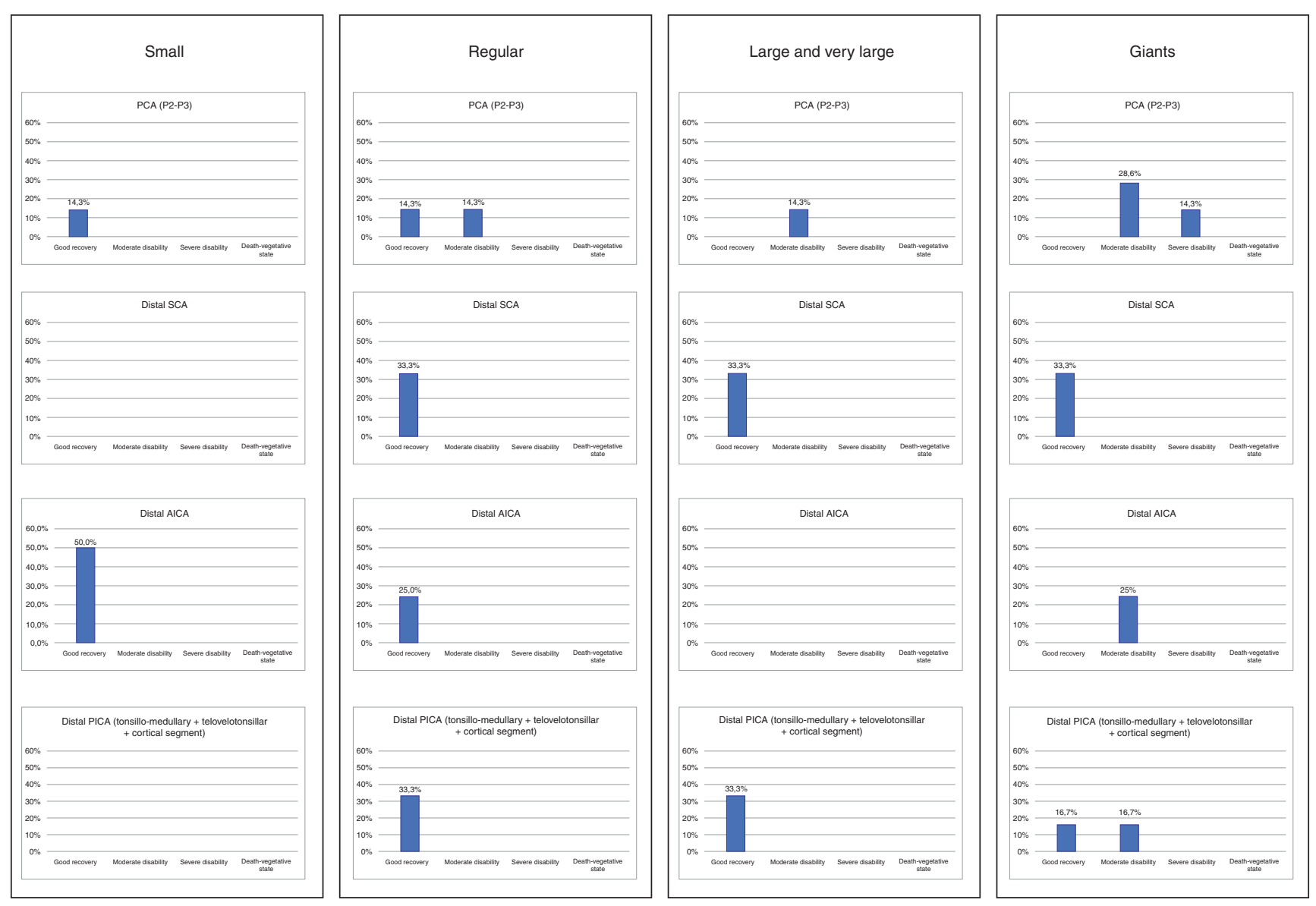

Fig. 2 Bar graph showing the overall patient outcome in distal aneurysms according their site and size

omy allows practical tailoring of any approach. The third remark regards the technological adjuvants, which are part of the surgeon's armamentarium. For instance, our group has already stressed the importance of endoscopeassisted techniques in the treatment of several neurosurgical pathologies, but particularly aneurysm surgery where, often, the endoscope view prevents perforating branches within blind spots $[25,26]$. Noteworthy, apart from the aforementioned aspects, a constant microneurosurgical training remains essential for aneurysms surgery, as already reported by our group [27].

The results of the present series confirm that microneurosurgery continues to have a paramount role in the treatment of many posterior circulation aneurysms, especially in young patients. In experienced hands, direct clipping allows for a definitive and durable exclusion of the aneurysm. Microneurosurgery also leads to a flow replacement before trapping for those aneurysms not amenable to coiling, stenting, or clipping.

Acknowledgments We want to thank Eng. Giorgia Di Giusto for the outstanding and continuous technical support.

Ethical Approval This study was approved by the Internal Advisory Board.

Conflict of Interest Statement The authors declare that they have no conflict of interest. 

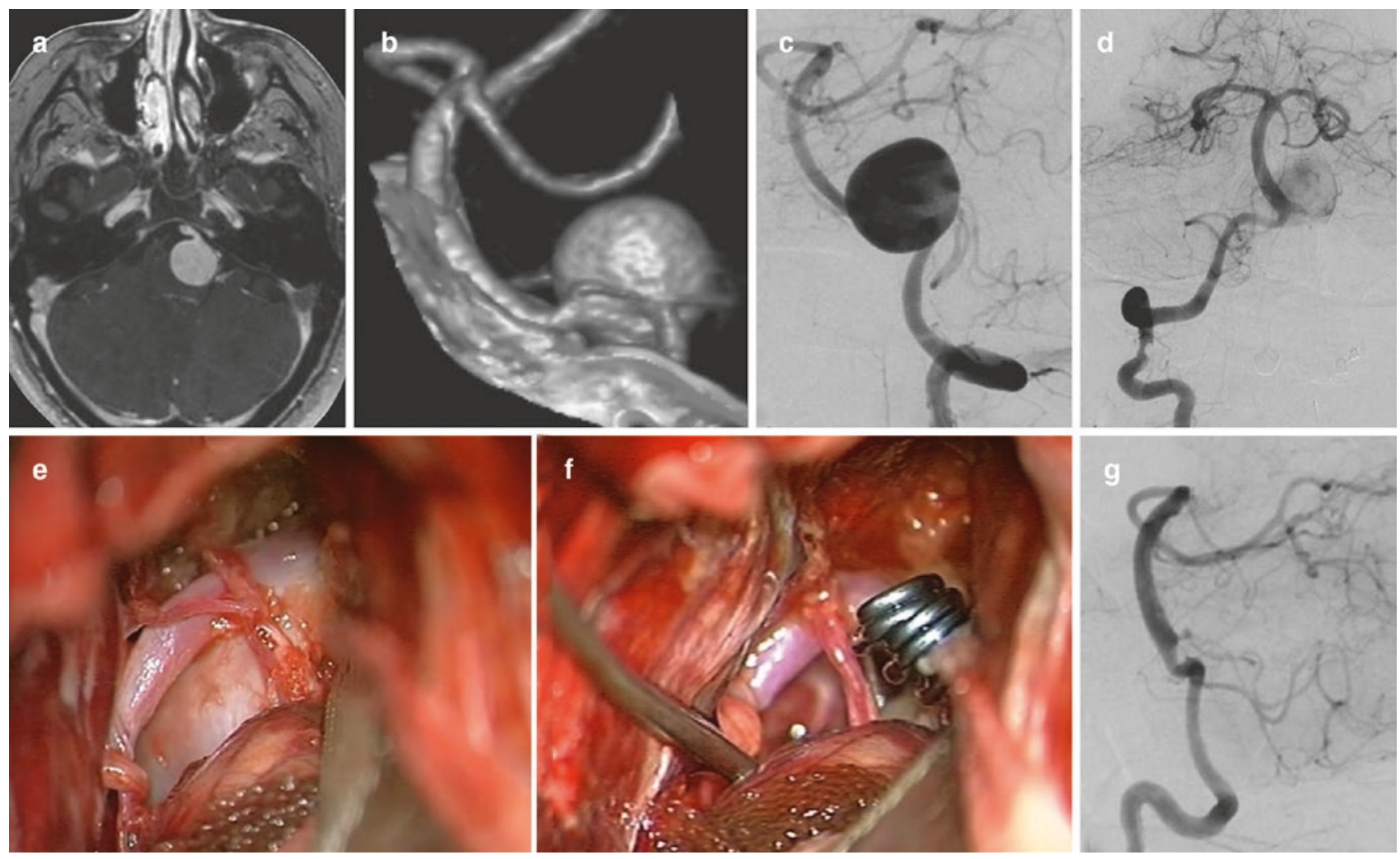

Fig. 3 Contrast-enhanced MRI showing a left giant VA causing a brainstem compression (a). CT angiography and DSA demonstrating the involvement of PICA (b, c). BTO revealing no crossflow (d). Left

\section{References}

1. Wiebers DO, Whisnant JP, Huston J, Meissner I, Brown RD, Piepgras DG, Forbes GS, Thielen K, Nichols D, O'Fallon WM, Peacock J, Jaeger L, Kassell NF, Kongable-Beckman GL, Torner JC, Investigators ISoUIA (2003) Unruptured intracranial aneurysms: natural history, clinical outcome, and risks of surgical and endovascular treatment. Lancet 362:103-110

2. Luzzi S, Elia A, Del Maestro M, Morotti A, Elbabaa SK, Cavallini A, Galzio R (2019) Indication, timing, and surgical treatment of spontaneous intracerebral hemorrhage: systematic review and proposal of a management algorithm. World Neurosurg. https://doi org/10.1016/j.wneu.2019.01.016

3. Al-Mefty O, Fox JL, Smith RR (1988) Petrosal approach for petroclival meningiomas. Neurosurgery 22:510-517

4. Luzzi S, Del Maestro M, Bongetta D, Zoia C, Giordano AV, Trovarelli D, Raysi Dehcordi S, Galzio RJ (2018) Onyx embolization before the surgical treatment of grade III Spetzler-Martin brain arteriovenous malformations: single-center experience and technical nuances. World Neurosurg 116:e340-e353. https://doi. org/10.1016/j.wneu.2018.04.203

5. Del Maestro M, Luzzi S, Gallieni M, Trovarelli D, Giordano AV, Gallucci M, Ricci A, Galzio R (2018) Surgical treatment of arteriovenous malformations: role of preoperative staged embolization. Acta Neurochir Suppl 129:109-113. https://doi. org/10.1007/978-3-319-73739-3_16

6. Luzzi S, Gallieni M, Del Maestro M, Trovarelli D, Ricci A, Galzio $\mathrm{R}$ (2018) Giant and very large intracranial aneurysms: surgical far-lateral retrocondylar approach and clipping of the aneurysm $(\mathbf{e}, \mathbf{f})$. Postoperative DSA documenting the complete exclusion of the aneurysm with a preserved flow into the left PICA (g)

strategies and special issues. Acta Neurochir Suppl 129:25-31. https://doi.org/10.1007/978-3-319-73739-3_4

7. Luzzi S, Del Maestro M, Elbabaa SK, Galzio R (2020) Letter to the Editor Regarding "One and Done: Multimodal Treatment of Pediatric Cerebral Arteriovenous Malformations in a Single Anesthesia Event". World Neurosurg 134:660. https://doi. org/10.1016/j.wneu.2019.09.166

8. Luzzi S, Del Maestro M, Galzio R (2019) Letter to the Editor. Preoperative embolization of brain arteriovenous malformations. J Neurosurg:1-2. https://doi.org/10.3171/2019.6.JNS191541

9. Luzzi S, Gragnaniello C, Giotta Lucifero A, Del Maestro M, Galzio R (2020) Microneurosurgical management of giant intracranial aneurysms: Datasets of a twenty-year experience. Data Brief 33:106537. https://doi.org/10.1016/j.dib.2020.106537

10. Luzzi S, Gragnaniello C, Giotta Lucifero A, Del Maestro M, Galzio R (2020) Surgical Management of Giant Intracranial Aneurysms: Overall Results of a Large Series. World Neurosurg. https://doi. org/10.1016/j.wneu.2020.08.004

11. Luzzi S, Gragnaniello C, Giotta Lucifero A, Stefano M, Elsawaf Y, Del Maestro M, Elbabaa SK, Galzio R (2020) Anterolateral Approach for Subaxial Vertebral Artery Decompression in The Treatment of Rotational Occlusion Syndrome: Results of A Personal Series and Technical Note. Neurological Research:1-16. https://doi.org/10.1080/01616412.2020.1831303

12. Luzzi S, Gragnaniello C, Marasco S, Lucifero AG, Del Maestro M, Bellantoni G, Galzio R (2020) Subaxial Vertebral Artery Rotational Occlusion Syndrome: An Overview of Clinical Aspects, Diagnostic Work-Up, and Surgical Management. Asian Spine J. https://doi. org/10.31616/asj.2020.0275 
13. Ricci A, Di Vitantonio H, De Paulis D, Del Maestro M, Raysi SD, Murrone D, Luzzi S, Galzio RJ (2017) Cortical aneurysms of the middle cerebral artery: A review of the literature. Surg Neurol Int 8:117. https://doi.org/10.4103/sni.sni_50_17

14. Rodriguez-Hernandez A, Walcott BP, Birk H, Lawton MT (2017) The superior cerebellar artery aneurysm: a posterior circulation aneurysm with favorable microsurgical outcomes. Neurosurgery 80:908-916. https://doi.org/10.1093/neuros/nyw111

15. Sanai N, Tarapore P, Lee AC, Lawton MT (2008) The current role of microsurgery for posterior circulation aneurysms: a selective approach in the endovascular era. Neurosurgery 62:1236-1249; discussion 1249-53. https://doi.org/10.1227/01. neu.0000333295.59738.de

16. Rodriguez-Hernandez A, Zador Z, Rodriguez-Mena R, Lawton MT (2013) Distal aneurysms of intracranial arteries: application of numerical nomenclature, predilection for cerebellar arteries, and results of surgical management. World Neurosurg 80:103-112. https://doi.org/10.1016/j.wneu.2012.09.010

17. Mascitelli JR, Lawton MT, Hendricks BK, Nakaji P, Zabramski JM, Spetzler RF (2019) Analysis of wide-neck aneurysms in the barrow ruptured aneurysm trial. Neurosurgery 85:622. https://doi. org/10.1093/neuros/nyy439

18. Krisht AF, Krayenbuhl N, Sercl D, Bikmaz K, Kadri PA (2007) Results of microsurgical clipping of 50 high complexity basilar apex aneurysms. Neurosurgery 60:242-250; discussion 250-2. https://doi.org/10.1227/01.NEU.0000249265.88203.DF

19. Nanda A, Sonig A, Banerjee AD, Javalkar VK (2014) Microsurgical management of basilar artery apex aneurysms: a single surgeon's experience from Louisiana State University, Shreveport. World Neurosurg 82:118-129. https://doi.org/10.1016/j. wneu.2013.06.016

20. Sekhar LN, Tariq F, Morton RP, Ghodke B, Hallam DK, Barber J, Kim LJ (2013) Basilar tip aneurysms: a microsurgical and endovascular contemporary series of 100 patients. Neurosurgery
72:284-298; discussion NEU.0b013e3182797952

21. Lehto H, Niemela M, Kivisaari R, Laakso A, Jahromi BR, Hijazy F, Andrade-Barazarte H, Dashti R, Hernesniemi J (2015) Intracranial vertebral artery aneurysms: clinical features and outcome of 190 patients. World Neurosurg 84:380-389. https://doi.org/10.1016/j. wneu.2015.03.034

22. Lehto H, Harati A, Niemela M, Dashti R, Laakso A, Elsharkawy A, Satopaa J, Billon-Grand R, Canato B, Kivisaari R, Hernesniemi J (2014) Distal posterior inferior cerebellar artery aneurysms: clinical features and outcome of 80 patients. World Neurosurg 82:702713. https://doi.org/10.1016/j.wneu.2014.06.012

23. Steven DA, Lownie SP, Ferguson GG (2007) Aneurysms of the distal anterior cerebral artery: results in 59 consecutively managed patients. Neurosurgery 60:227-233; discussion 234. https://doi. org/10.1227/01.NEU.0000249267.33945.E7

24. Zhang Y, Sun S, Xu Q, Feng W, Chen H (2018) Posterior inferior cerebellar artery aneurysm: have you ever been misdiagnosed? Acad Radiol 25:1564-1567. https://doi.org/10.1016/j.acra.2018.01.025

25. Gallieni M, Del Maestro M, Luzzi S, Trovarelli D, Ricci A, Galzio R (2018) Endoscope-assisted microneurosurgery for intracranial aneurysms: operative technique, reliability, and feasibility based on 14 years of personal experience. Acta Neurochir Suppl 129:19-24. https://doi.org/10.1007/978-3-319-73739-3_3

26. Arnaout MM, Luzzi S, Galzio R, Aziz K (2020) Supraorbital keyhole approach: Pure endoscopic and endoscope-assisted perspective. Clin Neurol Neurosurg 189:105623. https://doi.org/10.1016/j. clineuro.2019.105623

27. Del Maestro M, Rampini Angela D, Mauramati S, Giotta Lucifero A, Bertino G, Occhini A, Benazzo M, Galzio R, Luzzi S (2020) Dye-Perfused Human Placenta for Vascular Microneurosurgery Training: Preparation Protocol and Validation Testing. World Neurosurg. https://doi.org/10.1016/j.wneu.2020.11.034

Open Access This chapter is licensed under the terms of the Creative Commons Attribution 4.0 International License (http://creativecommons. org/licenses/by/4.0/), which permits use, sharing, adaptation, distribution and reproduction in any medium or format, as long as you give appropriate credit to the original author(s) and the source, provide a link to the Creative Commons license and indicate if changes were made.

The images or other third party material in this chapter are included in the chapter's Creative Commons license, unless indicated otherwise in a credit line to the material. If material is not included in the chapter's Creative Commons license and your intended use is not permitted by statutory regulation or exceeds the permitted use, you will need to obtain permission directly from the copyright holder.

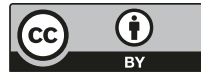

